\title{
Social Disadvantage, Access to Care, and Disparities in Physical Functioning Among Children Hospitalized with Respiratory Illness
}

\author{
Arti D Desai, MD, MSPH ${ }^{1,2 \star}$, Chuan Zhou, $\mathrm{PhD}^{1,2}$, Wren Haaland, MPH², Jakobi Johnson ${ }^{3}$, \\ K Casey Lion, MD, MPH',2, Michelle A Lopez, MD, MPH ${ }^{4}$, Derek J Williams MD, MPH ${ }^{3}$, Chén C Kenyon, MD, MSHP5, \\ Rita Mangione-Smith, MD, MPH',2, David P Johnson, $\mathrm{MD}^{3}$
}

\begin{abstract}
${ }^{1}$ Department of Pediatrics, University of Washington, Seattle, Washington; ${ }^{2}$ Seattle Children's Research Institute, Seattle, Washington; ${ }^{3}$ Division of Hospital Medicine, Monroe Carell Jr. Children's Hospital at Vanderbilt, Department of Pediatrics, Vanderbilt University School of Medicine, Nashville, Tennessee; ${ }^{4}$ Department of Pediatrics, Baylor College of Medicine, Houston, Texas; ${ }^{5}$ Center for Pediatric Clinical Effectiveness, The Children's Hospital of Philadelphia and the Department of Pediatrics, University of Pennsylvania School of Medicine, Philadelphia, Pennsylvania.
\end{abstract}

BACKGROUND AND OBJECTIVES: Understanding disparities in child health-related quality of life (HRQoL) may reveal opportunities for targeted improvement. This study examined associations between social disadvantage, access to care, and child physical functioning before and after hospitalization for acute respiratory illness.

METHODS: From July 1, 2014, to June 30, 2016, children ages 8-16 years and/or caregivers of children 2 weeks to 16 years admitted to five tertiary care children's hospitals for three common respiratory illnesses completed a survey on admission and within 2 to 8 weeks after discharge. Survey items assessed social disadvantage (minority race/ ethnicity, limited English proficiency, low education, and low income), difficulty/delays accessing care, and baseline and follow-up HRQoL physical functioning using the Pediatric Quality of Life Inventory (PedsQL, range 0-100). We examined associations between these three variables at baseline and follow-up using multivariable, mixed-effects linear regression models with multiple imputation sensitivity analyses for missing data.

RESULTS: A total of 1,325 patients and/or their caregivers completed both Peds $\mathrm{QL}$ assessments. Adjusted mean baseline PedsQL scores were significantly lower for patients with social disadvantage markers, compared with those of patients with none $(78.7$ for $>3$ markers versus 85.5 for no markers, difference -6.1 points $(95 \% \mathrm{Cl}:-8.7,-3.5)$. The number of social disadvantage markers was not associated with mean follow-up PedsQL scores. Difficulty/delays accessing care were associated with lower PedsOL scores at both time points, but it was not a significant effect modifier between social disadvantage and PedsOL scores.

CONCLUSIONS: Having social disadvantage markers or difficulty/delays accessing care was associated with lower baseline physical functioning; however, differences were reduced after hospital discharge. Journal of Hospital Medicine 2020;15:211-218. (C) 2020 Society of Hospital Medicine

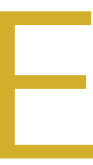

xamining disparities in health-related quality of life (HROoL) outcomes in children provides a unique patient-centered perspective on pediatric health services equity., ${ }^{1,2}$ Prior studies have demonstrated the relationship between minority race, low socioeconomic status, and lower maternal education with poorer HRQoL outcomes in children..$^{3-6}$ Some studies have also shown a dose-response relationship between social disadvantage markers and poorer child health status. ${ }^{7.8}$ Furthermore, the associations between social disadvantage and poor access to care, ${ }^{9-11}$ and between poor access to care and lower HRQoL, are also well established. ${ }^{12-14}$

\footnotetext{
*Corresponding Author: Arti D. Desai, MD, MSPH; E-mail: arti.desai@seattlechildrens.org; Telephone: (206) 884-1497.

Published online first February 19, 2020.

Find additional supporting information in the online version of this article.
}

Received: August 9, 2019; Revised: October 30, 2019;

Accepted: November 8, 2019

๑ 2020 Society of Hospital Medicine DOI 10.12788/jhm.3359
Examining HRQoL before and after hospitalization can further our understanding of how disparities in HRQoL may change once children engage with the medical system for an acute illness. ${ }^{15}$ Children requiring hospitalization constitute a useful population for examination of this question as they represent a group of children with variable social disadvantage markers and access to outpatient care. ${ }^{16}$ Although interventions to address social determinants of health for patients with social disadvantages have been associated with within-group improvements in HRQoL, none have assessed changes in disparities as an outcome. ${ }^{17}$ Furthermore, many of these studies were conducted in the outpatient setting, ${ }^{18,19}$ whereas hospitalization provides an additional point of care to address the social determinants of health for vulnerable families. ${ }^{20}$ Even for short hospitalizations, the 24/7 nature of hospital care provides the opportunity for frequent interactions with clinicians, nurses, and support staff to clarify illness-related questions, discuss other health concerns and unmet needs, and connect with social services or community resources. These opportunities may be particularly important for families with a higher number of social disadvantage markers and even more beneficial to 
those with difficulty accessing needed care from their primary medical home.

In this study, we focused on children with common respiratory illnesses (asthma, bronchiolitis, and pneumonia), which constitute the majority of childhood hospitalizations. ${ }^{21}$ Additionally, we only focused on the child's physical functioning component of HRQoL because this component is most likely to improve after hospitalization for children with an acute respiratory illness. ${ }^{22}$ A prior study examining HRQoL before and after hospitalization demonstrated that most children return to and/or surpass their baseline physical functioning by 1 month after hospital discharge..$^{23}$

Our primary objective was to examine associations between several markers of social disadvantage, access to care, and child physical functioning before and after hospitalization for acute respiratory illness. Second, we aimed to understand if access to care (defined as perceived difficulty/delays getting care) acts as an independent predictor of improvement in physical functioning from baseline to follow-up and/or if it modifies the relationship between social disadvantage and improvement in physical functioning.

\section{METHODS}

\section{Study Design and Population}

This study was nested within a multicenter, prospective cohort study of children who were hospitalized for asthma, bronchiolitis, or pneumonia between July 2014 and June 2016 at one of five children's hospitals in the Pediatric Research in Inpatient Settings Network. ${ }^{24}$

We approached families for study participation within 72 hours of admission to the hospital using a standard protocol. Patients and their caregivers were eligible to participate in the study if the patient was 2 weeks to 16 years old and if the primary caregiver's preferred language for medical communication was either English or Spanish. Patients with chronic medical conditions (except asthma), with moderate to severe developmental delay, with a history of prematurity $<32$ weeks, or who received care in the intensive care unit were excluded. Patients could only participate in the study once.

The study team set out to enroll an even number of patients across all three conditions. If a patient's discharge diagnosis differed from their admission diagnosis (eg, from bronchiolitis to pneumonia), discharge diagnosis was used for condition group assignment. If the discharge diagnosis was not one of these three respiratory conditions, we excluded the patient from further analysis.

\section{Data Collection}

We collected data using two surveys. The first survey was administered within 72 hours of admission. This survey asked questions related to (1) caregiver-reported markers of social disadvantage, (2) caregiver perceptions of access to care, and (3) caregiver- and patient-reported assessments of physical functioning. The second survey was administered within 2 to 8 weeks after the patient's discharge and included a second assessment of physical functioning.

\section{Social Disadvantage}

Patients were considered to have a marker of social disadvantage if their caregiver reported (1) being of non-White race and/or Hispanic ethnicity, (2) primarily speaking a language other than English at home and not speaking English very well (ie, limited English proficiency), (3) attaining at most a high school or equivalent degree, or (4) having a $\leq \$ 30,000$ annual household income.

\section{Access to Care}

We used the following survey item from the 2009-2010 National Survey of Children with Special Health Care Needs ${ }^{25}$ to measure caregiver perceptions of access to care: "In the last six months, did you have any difficulties or delays getting care for your child because there were waiting lists, backlogs, or other problems getting an appointment?" We narrowed the original assessment time frame from 12 months to 6 months to provide a more proximal assessment of access in relation to the hospitalization.

\section{Child Physical Functioning}

We assessed child physical functioning using the physical functioning domain of the Pediatric Quality of Life Inventory (PedsQL) 4.0 Generic Core Scales and PedsQL Infant Scales, which have been validated for use in the inpatient setting. ${ }^{22}$ Caregivers completed one of these scales based on their child's age. Assenting patients 8 to 16 years old completed the self-report PedsQL 4.0 Generic Core Scales instrument. When completing the first PedsQL survey, caregivers and patients reflected on the previous month before their child (or they) became ill to obtain a baseline physical functioning assessment. ${ }^{23}$ When completing the second Peds $\mathrm{QL}$ survey, caregivers and patients reflected on the past 7 days to obtain a follow-up assessment.

All study procedures were approved by the Western Institutional Review Board (IRB) or the participating hospitals' IRB.

\section{Statistical Analysis}

Patients with no missing data for all four social disadvantage markers were categorized based on the number of markers they reported: none, one, two, or three or more markers. We combined patients with three and four social disadvantage markers into one group to maximize power for the analyses. We dichotomized the access to care variable and coded response options as "no difficulty/delays accessing care" if the caregiver chose "Never" and "any difficulty/delays accessing care" if they chose "Sometimes/Usually/Always."

For each patient-caregiver dyad, PedsQL items were scored using a standard method in which higher scores reflected better functioning. ${ }^{22}$ A single set of PedsQL scores was used for each patient-caregiver dyad. We used self-reported patient scores if the patient completed the PedsQL instrument; otherwise, we used proxy-reported caregiver scores. Intraclass correlations between child self-report and parent proxy-report demonstrate moderate to good agreement above age 8 years. ${ }^{26}$ We computed a change in the physical functioning 
score by subtracting the baseline score from the follow-up score. The minimal clinically important difference (MCID) for the PedsQL instrument is 4.5 points, which we used to identify clinically meaningful differences. ${ }^{13}$

Analysis of variance models were constructed to test for differences in mean baseline and follow-up Peds $\mathrm{QL}$ scores (dependent variable) between the following independent variables: (1) social disadvantage groups and (2) those who reported having any difficulty/delays accessing care compared with those who did not. Only patient-caregiver dyads with both baseline and follow-up assessments were included in these analyses. Mixed-effects linear regression models were constructed to identify clinically meaningful differences in Peds $Q L$ scores between groups (MCID $\geq 4.5$ ) with adjustment for patient age, gender, respiratory condition, days between surveys, and hospital site as fixed effects. Site-specific random effects were included to account for within-hospital clustering. A similarly adjusted mixed-effects linear regression model was constructed to examine whether having any difficulty/delays accessing care modified the association between social disadvantage and $\mathrm{Peds} \mathrm{QL}$ change scores (eg, an improvement from baseline to follow-up).

Because $17 \%$ of respondents had missing data for at least one social disadvantage marker, sensitivity analyses were conducted using multiple imputation to account for missing social disadvantage markers using chained equations. ${ }^{27}$ Sensitivity analyses were also conducted to adjust for severity of illness using vital sign data within the first 24 hours, which could only be validly captured on patients with asthma within our dataset. By restricting this latter analysis to patients with asthma, we were able to examine the relationships of interest in a population with chronic disease.

\section{RESULTS}

The study sample included 1,860 patients, of which 1,325 had both baseline and follow-up PedsQL data (71\%). Descriptive statistics were similar between those who completed the baseline and follow-up surveys (Table 1).

Twenty-two percent of patients had $\geq 3$ social disadvantages and $30 \%$ of caregivers reported having any difficulty/delays accessing care. The mean follow-up Peds QL score was higher than the baseline score (90.4 vs 82.5; Table 1).

\section{Social Disadvantage Markers and PedsQL Scores}

The number of social disadvantage markers was inversely related to mean baseline PedsOL scores, but there was no difference in mean follow-up PedsOL scores between social disadvantage groups (Table 2). In adjusted analyses, the mean baseline PedsOL score was -6.1 points $(95 \% \mathrm{Cl}$ : $-8.7,-3.5)$ lower for patients with $\geq 3$ social disadvantage markers compared with patients with no social disadvantage markers, which exceeded the scale's MCID.

Difficulty/Delays Accessing Care and PedsQL Scores Having any difficulty/delays accessing care was significantly associated with lower baseline and follow-up PedsQL scores (Ta- ble 2). In adjusted analyses, the difference in baseline scores was 5.2 points ( $95 \% \mathrm{Cl}:-7.2,-3.2$ ), which exceedes the scale's MCID.

Interaction Between Social Disadvantage Markers, Difficulty/Delays Accessing Care, and Change in PedsQL Scores from Baseline to Follow-up

While having $\geq 2$ social disadvantage markers and difficulty/ delays accessing care were each positively associated with changes in PedsOL scores from baseline to follow-up (Table 3 ), only patients with $\geq 3$ social disadvantage markers exceeded the PedsQL MCID. In stratified analyses, patients with a combination of social disadvantage markers and difficulties/ delays accessing care had lower baseline PedsOL scores and greater change in PedsOL scores from baseline to follow-up compared with those without difficulties/delays accessing care (Figure). However, having any difficulty/delays accessing care did not significantly modify the relationship between social disadvantage and change in PedsOL scores, as none of the interaction terms were significant (Table 3, Model 3).

\section{Sensitivity Analysis}

Baseline, follow-up, and change in Peds $\mathrm{QL}$ scores were similar to our main analysis after performing multiple imputation for missing social disadvantage markers. Findings were also similar for patients with a diagnosis of asthma only; however, changes in PedsQL scores were greater in magnitude.

\section{DISCUSSION}

This study examined the relationship between social disadvantage and child physical functioning before and after hospitalization for acute respiratory illness. Study findings indicated that patients with higher numbers of social disadvantage markers reported lower Peds $\mathrm{QL}$ scores before hospitalization; however, differences in PedsOL scores were not apparent after hospitalization. Patients who experienced difficulty/delays accessing care also reported lower PedsOL scores at baseline. This difference was still significant but did not exceed the Peds QL MCID threshold after hospitalization. Difficulty/delays accessing care appeared to be an additional social disadvantage marker; however, it did not modify the relationship between social disadvantage and improvement in physical functioning.

The study findings at baseline are consistent with prior studies demonstrating a negative association between social disadvantage markers and $\mathrm{HRQ} \mathrm{L}$ and a cumulative effect based on the number of social disadvantages.,3,7,8 This study adds to the existing literature by examining how this relationship changes after hospitalization. As evidenced by the lack of association between social disadvantage markers and follow-up $\mathrm{Peds} \mathrm{QL}$ scores, our findings suggest that receipt of inpatient care improved perceptions of physical functioning to a greater extent for patients with more social disadvantage markers (especially patients with $\geq 3$ social disadvantage markers). There are several potential reasons for these findings.

One possibility is that caregivers and/or patients with more social disadvantage markers are more influenced by context 
TABLE 1. Patient Demographics and Descriptive Statistics

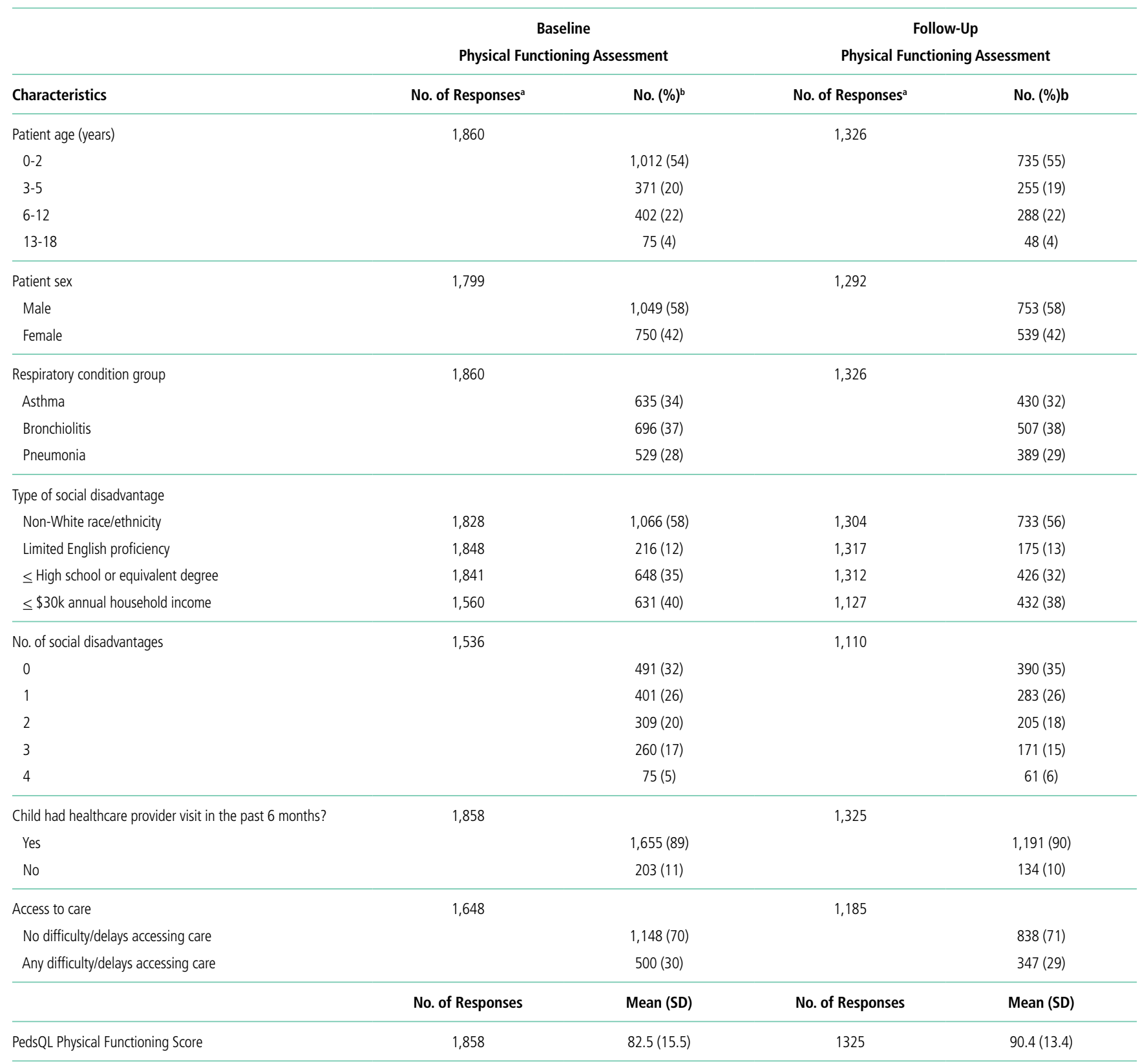

aThe number of observations in each category diverges from the total number in full cohort because of missing data.

bProportions may diverge from $100 \%$ due to rounding.

Abbreviations: PedsQL, Pediatric Quality of Life Inventory; SD, standard deviation.

when assessing physical functioning. This could lead to an underestimation of functioning when asked to recall baseline physical functioning at the time of acute illness and overestimation of functioning after recovery from an illness. This possibility is consistent with a form of response bias, extreme response tendencies, in which lower socioeconomic subgroups tend to choose the more extreme response options of a scale. ${ }^{28}$ In the absence of longitudinal assessments of HRQOL across the care continuum over time, disentangling whether these differences are due to response bias or representative of true changes in physical functioning remains challenging.

Given that disparities in physical functioning at baseline were consistent with prior evidence, another possibility is that hospitalization provided an opportunity to address gaps in access and quality that may have existed for patients with social disadvantage in the community setting. The 24/7 nature of hospital care, usually from a multidisciplinary team of providers, lends itself to opportunities to receive intensive education related to the current illness or to address other health concerns that parents or providers identify during a hospital stay. 
TABLE 2. Observed Means for PedsQL Physical Functioning Scores by Number of Social Disadvantage Markers and Difficulty/Delays Accessing Outpatient Care Before and After Receiving ED/Hospital Care

\begin{tabular}{|c|c|c|c|c|}
\hline & & $\begin{array}{l}\text { Baseline PedsQL } \\
\text { Physical Functioning Score }\end{array}$ & $\begin{array}{l}\text { Follow-up PedsQL } \\
\text { Physical Functioning Score }\end{array}$ & $\begin{array}{l}\text { Change in PedsQL } \\
\text { Physical Functioning Score }\end{array}$ \\
\hline No. of Social Disadvantages & No. ${ }^{a}$ & Mean (SD) & Mean (SD) & Mean (SD) \\
\hline 0 & 390 & $85.5(12.9)$ & $90.4(12.9)$ & $+4.9(15.6)$ \\
\hline 1 & 283 & $83.6(14.2)$ & $90.0(13.5)$ & $+6.5(16.3)$ \\
\hline$\geq 3$ & 232 & $78.7(17.9)^{\mathrm{b}}$ & $90.9(13.8)$ & $+12.2(17.2)$ \\
\hline Overall & 1,110 & $83.0(15.1)$ & $90.8(13.3)$ & $+7.5(16.4)$ \\
\hline ANOVA $P$ value & & $<.001$ & .91 & $<.001$ \\
\hline \multicolumn{5}{|l|}{ Difficulty/Delays Accessing Care } \\
\hline Overall & 1,185 & $82.2(15.3)$ & $90.2(13.6)$ & $+8.0(16.7)$ \\
\hline ANOVA $P$ value & & $<.001$ & $<.01$ & $<.01$ \\
\hline \multicolumn{5}{|c|}{$\begin{array}{l}\text { anly includes patients who completed both the baseline and follow-up PedsQL assessments. } \\
\text { bDifference in score from the score of those with no social disadvantage markers exceeded the PedsQL minimal clinically important difference of } 4.5 \text { points. } \\
\text { 'Difference in score from the score of those with no difficulty/delays accessing care exceeded the PedsQL minimal clinically important difference of } 4.5 \text { points. } \\
\text { Abbreviations: ANOVA, analysis of variance; PedsQL, Pediatric Quality of Life Inventory; SD, standard deviation. }\end{array}$} \\
\hline
\end{tabular}

TABLE 3. Adjusted Associations Between Social Disadvantage, Difficulty/Delay Accessing Outpatient Care and Change in PedsQL Physical Functioning Scores from Baseline to Follow-up

\begin{tabular}{|c|c|c|c|}
\hline \multirow[t]{2}{*}{ Predictor of interest } & Model $1^{\mathrm{a}}$ & Model $2^{\mathrm{a}}$ & Model $3^{a}$ \\
\hline & $\beta(95 \% \mathrm{Cl})^{\mathrm{b}}$ & $\beta(95 \% \mathrm{Cl})^{\mathrm{b}}$ & $\beta(95 \% \mathrm{Cl})^{b}$ \\
\hline \multicolumn{4}{|l|}{ No. of Social Disadvantages } \\
\hline 0 & Ref & & Ref \\
\hline$\geq 3$ & $6.1(4.6,7.7)^{* * *}$ & & $6.9(4.3,9.5)^{* * *}$ \\
\hline \multicolumn{4}{|l|}{ Difficulty/Delay Accessing Care } \\
\hline None & & Ref & Ref \\
\hline Any $D / D$ & & $2.4(0.2,4.6)^{*}$ & $2.9(2.2,3.6)^{* * *}$ \\
\hline \multicolumn{4}{|l|}{ Interaction } \\
\hline
\end{tabular}

$\star P<.05 ; * * P<.01 ; * \star * P<.001$.

aModels 1-3 are adjusted for patient age, gender, respiratory condition, days between surveys, hospital site, and clustering by site. Model 1 only had social disadvantage as the main effect; Model 2 only had difficulty/delays accessing care as the main effect; and Model 3 had social disadvantage, difficulty/delays accessing care, and their interaction as the main effects.

${ }^{b}$ Regression coefficients represent the difference in PedsQL change score from the reference category, where clinically meaningful differences are $>4.5$ points based on the minimal clinically important difference on the PedsQL instrument.

Abbreviations: $\beta$, regression coefficient; D/D, difficulty/delays accessing care; PedsQL, Pediatric Quality of Life Inventory. 


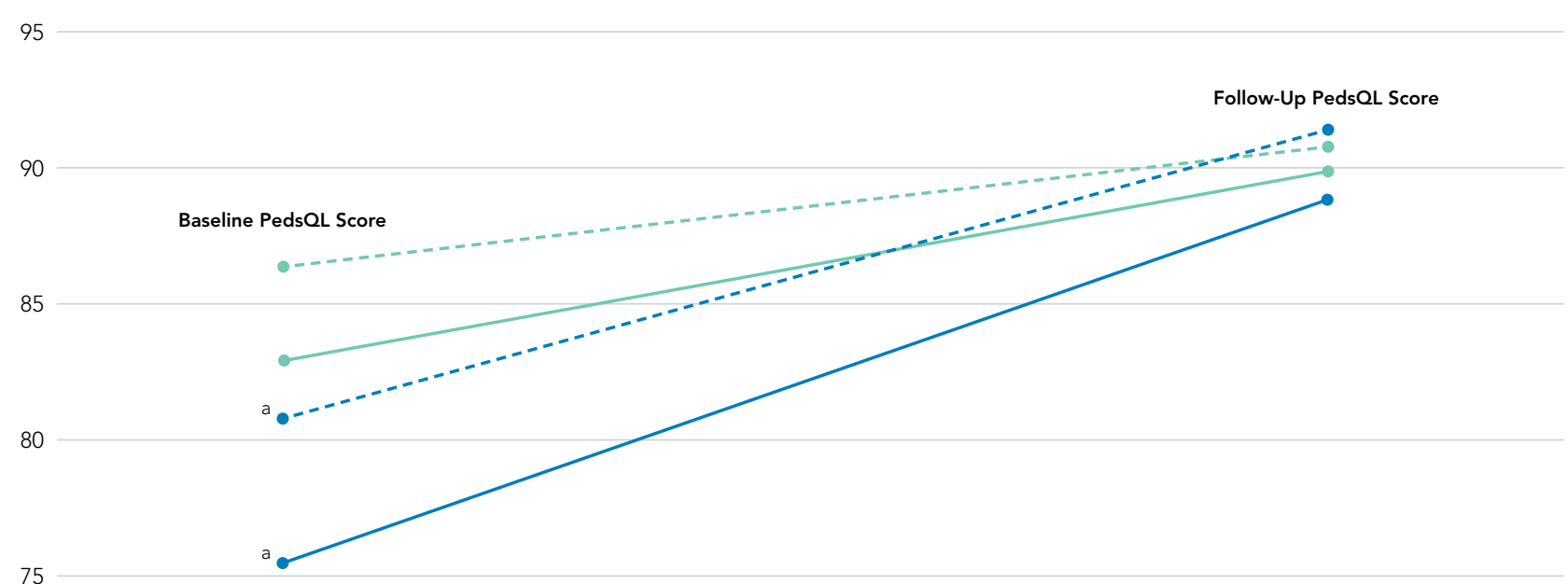

75

70

$\bullet---$ No SD \& No D/D $\longrightarrow$ No SD \& Any D/D $\longrightarrow---\bullet \geq 3$ SD \& No D/D 3 SD \& Any D/D

FIG. Observed Change in PedsQL Physical Functioning Scores from Baseline to Follow-up.

a Indicates the difference in PedsQL score from the reference score exceeded the PedsQL minimal clinically important difference of 4.5 points. Abbreviations: D/D, difficulties/delays accessing outpatient care; PedsQL, Pediatric Quality of Life Inventory; SD, social disadvantage markers.

For example, consistent and repetitive asthma education may be more beneficial to patients and families with more social disadvantage markers. The fact that the association between social disadvantage markers and change in physical functioning scores were greater for patients with asthma supports this reasoning. Hospital care may also provide an opportunity to address other unmet medical needs or psychosocial needs by providing efficient access to subspecialists, social workers, or interpreters. Further research is needed to elucidate whether families received additional services in the hospital setting that were not available to them prior to hospitalization, such as consistent interpreter use, social work engagement, and subspecialty/community referrals. Further studies should also determine whether the provision of equitable medical and social support services is associated with improvements in HRQoL disparities. Additionally, studies should examine whether physical functioning improvements following hospitalization return to baseline levels after a longer period of time and, if so, how we might sustain these reductions in HROoL disparities. Such studies may identify tangible targets and interventions to reduce disparities in HRQoL for these children.

This study highlights the importance of assessing for difficulty/delays accessing care in addition to social disadvantage markers, as this was also a significant predictor of lower child physical functioning. Differences in PedsQL scores between those who reported any versus no difficulty/delays accessing care were more pronounced at baseline compared with follow-up. A possible reason for these findings is that receiving hospital care may have addressed some access to care issues that were present in the outpatient setting, which resulted in improved perceptions of physical functioning. For example, hospital care may mitigate access to care barriers such as limited after-hours clinic appointments, language barriers, and lack of insurance, thus providing some patients with an alternative pathway to address their health concerns. Alternatively, hospital staff may assist families in scheduling follow-up appointments with the patient's primary medical home after discharge, which potentially reduced some access to care barriers. The question is whether these disparities will widen once again after a longer follow-up period if families continue facing barriers to accessing needed care in the outpatient setting.

The results of the effect modification analysis demonstrated that the association between social disadvantage and change in PedsQL scores from baseline to follow-up was not significantly different based on a child's ability to access care. In our stratified analysis, difficulty/delays accessing care added to baseline disparities at each social disadvantage level but did not alter how perceptions of physical functioning change over time. Therefore, physical functioning improvements may rely more heavily on the type of care received within the hospital setting as opposed to accessing care in the first place. However, future studies should examine whether access to highquality care instead of simply measuring difficulty/delays in accessing care would lead to different results. Access to a comprehensive medical home may be a better measure to assess for effect modification because it measures features beyond access to care, such as continuity, comprehensiveness, communication, and coordination of outpatient care. ${ }^{29-31}$ 
If additional studies find evidence that the nature of hospital care, an intensive 24/7 care setting, differentially benefits patients with higher social disadvantage markers (particularly those with $\geq 3$ markers and chronic illness), this would support the need for systematic screening for social disadvantages or difficulty/delays accessing care in the inpatient setting. Systematic screening could help ensure all patients who may benefit from additional services, such as intensive, culturally tailored education or connections to food, housing, or financial services, will in fact receive them, which may lead to sustained reductions in health disparities. ${ }^{20}$ Further research into pairing validated screening tools with proven interventions is needed. ${ }^{32}$

This study has additional limitations aside from those noted above. First, we did not reassess perceived or actual access to care after hospitalization, which may have allowed for analyses to examine access to care as a mediator between social disadvantage and lower child physical functioning. Second, this study included only English- and Spanish-speaking patients and families. Patients with less commonly spoken languages may experience more difficulty accessing or navigating the health system, which may further impact access to care and HRQoL. Third, we had a considerable amount of missing social disadvantage marker data (mainly income); however, our sensitivity analyses did not result in significantly different or clinically meaningful differences in our findings. Insurance status is more feasible to obtain from administrative data and could serve as a proxy for income or as an additional social disadvantage marker in future studies. Finally, we could calculate illness severity only for patients with asthma based on the available data; therefore, we could not adequately control for illness severity across all conditions.

\section{CONCLUSIONS}

Social disadvantage was associated with lower child physical functioning before hospitalization, but differences were not apparent after hospitalization for children with acute respiratory illness. Caregiver-perceived difficulty/delays accessing care was found to be an additional predictor of lower physical functioning at baseline but did not significantly alter the association between social disadvantage and improvement in physical functioning over time. Further studies are needed to understand how hospital care may differentially impact child physical functioning for patients with higher social disadvantage makers in order to sustain improvements in HRQoL disparities.

\section{Acknowledgments}

The authors thank the following individuals of the Pediatric Respiratory Illness Measurement System (PRIMES) study team for their contributions to this work: Karen M. Wilson, New York, New York; Ricardo A. Quinonez, Houston, Texas; Joyee G. Vachani, Houston, Texas; and Amy Tyler, Aurora, Colorado. We would also like to thank the Pediatric Research in Inpatient Settings Network for facilitating this work.

Disclosures: The authors have no financial relationships relevant to this article to disclose. The authors have no conflicts of interest relevant to this article to disclose.

Funding: Research reported in this article was supported by the National Heart, Lung, and Blood Institute of the National Institutes of Health under award num- ber R01HL121067-01 (PI: Rita Mangione-Smith). Dr. Desai's time was supported by Agency for Healthcare Research and Quality grant K08 HS024299 (PI Desai). Dr. Lion's time was supported by National Institute of Child Health and Human Development grant K23 HD078507 (PI Lion).

\section{References}

1. Szilagyi PG, Schor EL. The health of children. Health Serv Res. 1998;33(4 Pt 2):1001-1039.

2. Varni JW, Burwinkle TM, Lane MM. Health-related quality of life measurement in pediatric clinical practice: an appraisal and precept for future research and application. Health Qual Life Outcomes. 2005;3(1):34. https://doi. org/10.1186/1477-7525-3-34

3. von Rueden U, Gosch A, Rajmil L, Bisegger C, Ravens-Sieberer U. Socioeconomic determinants of health related quality of life in childhood and adolescence: results from a European study. J Epidemiol Community Health. 2006;60(2):130-135. https://doi.org/10.1136/jech.2005.039792.

4. Quittner AL, Schechter MS, Rasouliyan L, Haselkorn T, Pasta DJ, Wagener JS. Impact of socioeconomic status, race, and ethnicity on quality of life in patients with cystic fibrosis in the United States. Chest. 2010;137(3):642-650. https://doi.org/10.1378/chest.09-0345.

5. Flores G, Tomany-Korman SC, Corey CR, Freeman HE, Shapiro MF. Racial and ethnic disparities in medical and dental health, access to care, and use of services in US children. Pediatrics. 2008;121(2):e286-98. https://doi. org/10.1542/peds.2007-1243.

6. Fedele DA, Molzon ES, Eddington AR, Hullmann SE, Mullins LL, Gillaspy SG. Perceived barriers to care in a pediatric medical home: the moderating role of caregiver minority status. Clin Pediatr (Phila). 2014;53(4):351-355. https:// doi.org/10.1177/0009922813507994.

7. Larson K, Russ SA, Crall JJ, Halfon N. Influence of multiple social risks on children's health. Pediatrics. 2008;121(2):337-344. https://doi.org/10.1542/ peds.2007-0447

8. Bauman LJ, Silver EJ, Stein REK. Cumulative social disadvantage and child health. Pediatrics. 2006;117(4):1321-1328. https://doi.org/10.1542/ peds.2005-1647

9. Andrulis DP. Moving beyond the status quo in reducing racial and ethnic disparities in children's health. Public Health Rep. 2005;120(4):370-377. https:// doi.org/10.1177/003335490512000403.

10. Flores G, Lin H. Trends in racial/ethnic disparities in medical and oral health access to care, and use of services in US children: has anything changed over the years? Int J Equity Health. 2013;12:10. https://doi.org/10.1186/1475-9276-12-10.

11. Seid M, Stevens GD, Varni JW. Parents' perceptions of pediatric primary care quality: effects of race/ethnicity, language, and access. Health Serv Res. 2003;38(4):1009-1031. https://doi.org/10.1111/1475-6773.00160.

12. Seid M, Varni JW, Cummings L, Schonlau M. The impact of realized access to care on health-related quality of life: a two-year prospective cohort study of children in the California State Children's Health Insurance Program. J Pediatr. 2006;149(3):354-361. https://doi.org/10.1016/j.jpeds.2006.04.024.

13. Varni JW, Burwinkle TM, Seid M, Skarr D. The PedsQL 4.0 as a pediatric population health measure: feasibility, reliability, and validity. Ambul Pediatr. 2003;3(6):329-341. https://doi.xorg/10.1367/1539-4409(2003)003<0329:tpaapp $>2.0 . c 0 ; 2$.

14. Simon AE, Chan KS, Forrest CB. Assessment of children's health-related quality of life in the united states with a multidimensional index. Pediatrics. 2008;121(1):e118-e126. https://doi.org/10.1542/peds.2007-0480.

15. Cheng TL, Emmanuel MA, Levy DJ, Jenkins RR. Child health disparities: what can a clinician do? Pediatrics. 2015;136(5):961-968. https://doi.org/10.1542/ peds.2014-4126

16. Christakis DA, Mell L, Koepsell TD, Zimmerman FJ, Connell FA. Association of lower continuity of care with greater risk of emergency department use and hospitalization in children. Pediatrics. 2001;107(3):524-529. https://doi. org/10.1542/peds.107.3.524

17. Lion KC, Raphael JL. Partnering health disparities research with quality improvement science in pediatrics. Pediatrics. 2015;135(2):354-361. https://doi. org/10.1542/peds.2014-2982.

18. Williams DR, Costa MV, Odunlami AO, Mohammed SA. Moving upstream: how interventions that address the social determinants of health can improve health and reduce disparities. J Public Health Manag Pract. 2008;14:S8-S17. https://doi.org/10.1097/01.PHH.0000338382.36695.42.

19. Beck AF, Cohen AJ, Colvin JD, et al. Perspectives from the Society for Pediatric Research: interventions targeting social needs in pediatric clinical care. Pediatr Res. 2018;84(1):10-21. https://doi.org/10.1038/s41390-018-0012-1.

20. Shah AN, Simmons J, Beck AF. Adding a vital sign: considering the utility of place-based measures in health care settings. Hosp Pediatr. 2018;8(2):112- 
114. https://doi.org/10.1542/hpeds.2017-0219.

21. Leyenaar JK, Ralston SL, Shieh M-S, Pekow PS, Mangione-Smith R, Lindenauer PK. Epidemiology of pediatric hospitalizations at general hospitals and freestanding children's hospitals in the United States. J Hosp Med. 2016;11(11):743-749. https://doi.org/10.1002/jhm.2624.

22. Desai AD, Zhou C, Stanford S, Haaland W, Varni JW, Mangione-Smith RM Validity and responsiveness of the pediatric quality of life inventory (Peds $\mathrm{QL}$ ) 4.0 generic core scales in the pediatric inpatient setting. JAMA Pediatr. 2014;168(12):1114-1121. https://doi.org/10.1001/jamapediatrics.2014.1600.

23. Rabbitts JA, Palermo TM, Zhou C, Mangione-Smith R. Pain and health-related quality of life after pediatric inpatient surgery. J Pain. 2015;16(12):1334-1341. https://doi.org/10.1016/j.jpain.2015.09.005.

24. Mangione-Smith R, Zhou C, Williams DJ, et al. Pediatric respiratory illness measurement system (PRIMES) scores and outcomes. Pediatrics. 2019;144(2):e20190242. https://doi.org/10.1542/peds.2019-0242

25. Child and Adolescent Health Measurement Initiative. National survey of children with special health care needs (NS-CSHCN), 2009-2010. Available at: http://childhealthdata.org/learn/NS-CSHCN/topics_questions. Accessed on September 20, 2018.

26. Varni JW, Limbers CA, Burwinkle TM. How young can children reliably and validly self-report their health-related quality of life?: an analysis of 8,591 children across age subgroups with the PedsQL 4.0 Generic Core Scales. Health
Qual Life Outcomes. 2007;5:1. https://doi.org/10.1186/1477-7525-5-1.

27. Buuren S van, Groothuis-Oudshoorn K. Mice: Multivariate imputation by chained equations in R. J Stat Softw. 2011;45(3):1-67. https://doi. org/10.18637/jss.v045.i03.

28. Elliott MN, Haviland AM, Kanouse DE, Hambarsoomian K, Hays RD. Adjusting for subgroup differences in extreme response tendency in ratings of health care: impact on disparity estimates. Heal Serv Res. 2009;44(2 Pt 1):542561. https://doi.org/10.1111/j.1475-6773.2008.00922.x

29. Stevens GD, Vane C, Cousineau MR. Association of experiences of medical home quality with health-related quality of life and school engagement among Latino children in low-income families. Health Serv Res. 2011;46(6pt1):1822-1842. https://doi.org/10.1111/j.1475-6773.2011.01292.x.

30. Long WE, Bauchner H, Sege RD, Cabral HJ, Garg A. The value of the medical home for children without special health care needs. Pediatrics. 2012;129(1):87-98. https://doi.org/10.1542/peds.2011-1739.

31. Strickland BB, Jones JR, Ghandour RM, Kogan MD, Newacheck PW. The medical home: health care access and impact for children and youth in the United States. Pediatrics. 2011;127(4):604-611. https://doi.org/10.1542/ peds.2009-3555.

32. Sokol R, Austin A, Chandler C, et al. Screening children for social determinants of health: a systematic review. Pediatrics. 2019;144(4):e20191622. https://doi.org/10.1542/peds.2019-1622. 